\title{
Monte Carlo Simulation Experiments for Engineering Optimisation
}

\author{
Robert Anderson ${ }^{1}$, Zhou $\mathrm{Wei}^{2}$, Ian $\mathrm{Cox}^{1}$, Malcolm Moore ${ }^{1}$, Florence Kussener ${ }^{3}$ \\ ${ }^{1}$ SAS Institute, Marlow, UK \\ ${ }^{2}$ SAS Institute, Beijing, China \\ ${ }^{3}$ SAS Institute, Gregy sur Yerre, France \\ Correspondence: Robert Anderson, SAS Institute, Marlow, UK
}

Received: June 25, 2015 Accepted: July 7, 2015 Online Published: July 22, 2015

doi:10.11114/set.v2i1.901 URL: http://dx.doi.org/10.11114/set.v2i1.901

\begin{abstract}
Design of Experiments (DoE) is widely used in design, manufacturing and quality management. The resulting data is usually analysed with multiple linear regression to generate polynomial equations that describe the relationship between process inputs and outputs. These equations enable us to understand how input values affect the predicted value of one or more outputs and find good set points for the inputs. However, to develop robust manufacturing processes, we also need to understand how variation in these inputs appears as variation in the output. This understanding allows us to define set points and control tolerances for the inputs that will keep the outputs within their required specification windows. Tolerance analysis provides a powerful way of finding input settings and ranges that minimise output variation to produce a process that is robust. In many practical applications, tolerance analysis exploits Monte Carlo simulation of the polynomial model generated from DoE's. This paper briefly describes tolerance analysis and then shows how Monte Carlo simulation experiments using space-filling designs can be used to find the input settings that result in a robust process. Using this approach, engineers can quickly and easily identify the key inputs responsible for transferring undesired variation to their process outputs and identify the set points and ranges that make their process as robust as possible. If the process is not sufficiently robust, they can rationally investigate different strategies to improve it. A case study approach is used to aid explanation and understanding.
\end{abstract}

Keywords: engineering optimisation, tolerance analysis, quality by design, QBD, Monte Carlo simulation, simulation experiment, Gaussian process, definitive screening designs

\section{Introduction}

Design of Experiments (DoE) is often used to identify the important factors and model the effects of these factors using linear polynomial equations including a noise term for the unexplained variation. See Moore, Anderson and Kay (2015) for a discussion of the benefits of DoE and the types of scientific and engineering problems that can be solved with DoE. Typically, screening DoE's, such as full or fractional factorials, or D-optimal designs are used to identify the important factors and screen out unimportant factors by modelling main effects (linear terms) and, occasionally, two-way interaction effects (cross terms from two linear terms). Response Surface Modelling (RSM) designs are then used to develop the model to identify any active higher order (quadratic and two-way interaction) terms. The RSM model obtained is then used to find settings of the factors that will result in the desired target value(s) for the output(s). Readers new to DoE can find more details of the methods along with examples in Box, Hunter, and Hunter (2005); Kenett and Zacks (2014); Montgomery (2012); and Goos and Jones (2011). As explained by Moore et al (2015), the DoE method is continually evolving with a new class of screening designs being proposed by Jones and Nachtsheim (2011) called definitive screening - a three level screening design. They have a practical appeal to engineers, because unlike some classical screening designs, they don't confound any of the main effects with any of the two-way interactions or quadratic terms. In situations where only three or fewer of the main effects are active, these designs collapse into full RSM designs and allow any active two-way interaction terms or quadratic terms to be modelled. The use of definitive screening designs can now often allow an RSM model to be estimated via a very efficient single stage experiment.

In the past, Taguchi methods have been used to simultaneously optimize the mean and variance of a response using an inner array for the control factors and an outer array for the noise factors. Unfortunately with this approach, the size of the experiment required rapidly becomes very large. Monte Carlo simulation with definitive screening designs provides 
a more practical alternative, and allows processes to be modelled and optimised with respect to output mean and variation using much smaller experimental budgets. In a previous paper by Wei, Moore and Kussener (2013), visual tolerance analysis (based on Monte Carlo techniques) was shown to be an effective way of finding input settings that reduce output variation. This paper shows how to usefully extend their approach using a space-filling simulation experiment to more systematically explore the region defined by the inputs. The main objective of this study being to illustrate the efficiency and effectiveness of this simulation experiment approach.

\section{Propagation of Error}

Design of Experiments (DoE) and statistical or mechanistic modelling are widely used to help create and develop new products and processes and then to transfer these into production. In order to successfully transfer a product or process to production, it is necessary to have an understanding of how consistently the product or process will meet the required quality or performance characteristics. In other words, we need to estimate how often the product or process will comply with all of the required specification limits. Any product or process material that does not comply will be deemed to be defective and may have to be reworked or scrapped. Clearly, to maximise profit and minimise costs, the level of defective output has to be minimised. Often, when large-scale production begins, the level of variation and defective product turns out to be larger than first anticipated. Frequently, the actual process capability is much poorer than indicated from small-scale R\&D trials. This invariably results in additional engineering overhead to incrementally improve process capability after the product or process has gone into full-scale manufacturing. Techniques that can better identify the sources of process and product variation and optimise these to make the product or process more robust once it is in full manufacturing, offer substantial cost benefits.

\section{Tolerance Analysis and Monte Carlo Simulation Experiments}

The relentless demand for higher and higher levels of quality but at lower cost, has led to the development of advanced techniques such as tolerance analysis and Quality by Design (QbD). The KPA group (2015) provide a concise overview of $\mathrm{QbD}$ and explain how it has played a vital role in the pharmaceutical drug development and approval process. Tolerance analysis is a related approach that models the propagation of any variation in the inputs through to the resulting variation in the outputs. This allows control tolerances to be defined for all the critical input variables during R\&D, rather than simply defining set points. This ensures that the outputs are insensitive to variation in inputs, guaranteeing that customer requirements and product specification limits will be met. In practice, using the widest input control tolerances consistent with producing acceptable levels of variation in the outputs will reduce costs considerably, since tighter control tolerances are always more expensive and may not even be achievable in manufacturing.

\section{Method}

A case study approach will be used to demonstrate the Monte Carlo simulation experiment method for tolerance analysis in engineering optimisation. Rubinstein and Kroese (2008) and http://en.wikipedia.org/wiki/Monte_Carlo_method (2015) provide a detailed description and good overview of the Monte Carlo simulation approach and methods.

\subsection{Background}

The example is based on a 13-run definitive screening design (DSD) with five factors and one response. These three-level definitive screening designs are a new class of screening designs that can efficiently allow you to identify the important main effects. Unlike fractional-factorial screening designs or Plackett-Burman screening designs, they don't confound or alias any of the main effects with any of the two-way interactions. Since they are three-level designs, they can also estimate quadratic terms and those quadratic terms are not confounded or aliased with any of the main effects either. These designs are reasonably small and efficient as they only require $2 n+1$ runs where $n$ is the number of factors and $n$ is even and $2 n+3$ runs when $n$ is odd. When there are only three or fewer active main effects, they allow a full response surface model with two-factor interactions and quadratic terms to be modelled. Table 1 shows the 13-run definitive screening design in five factors as a JMP table. JMP is Statistical Discovery software from SAS that was used to create the definitive screening design and analyse this case study. 
Table 1. Experimental schedule and results

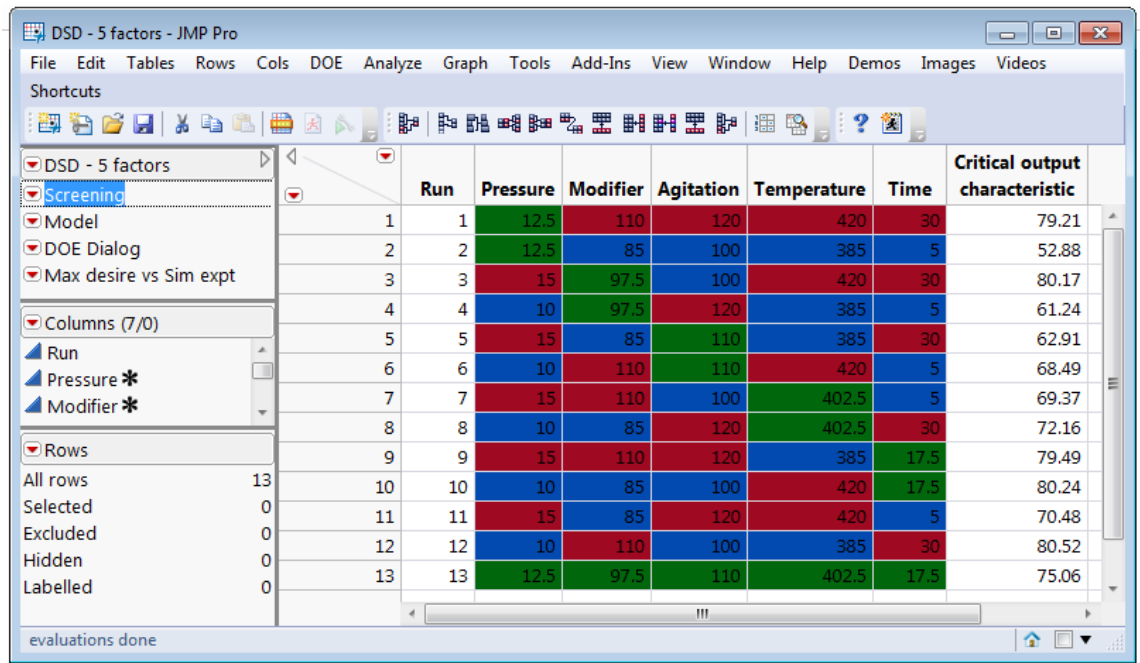

\subsection{Monte Carlo Simulation Process}

In this case study, the critical output characteristic is a yield (called "Critical output characteristic") that needs to be maximised and must be greater than 75 percent. After estimation and selection of a "best" regression model by standard least square method, we obtain a model that contains three main effects; Modifier, Temperature and Time plus a two-factor interaction term between Modifier and Temperature and a quadratic term for Time. The JMP prediction profiler for this model is shown in figure 1. The Monte Carlo simulator and the contour profiler are also shown. With this model, Monte Carlo simulation can be used to assess the extent to which variation in the inputs propagates through to variation in the outputs.

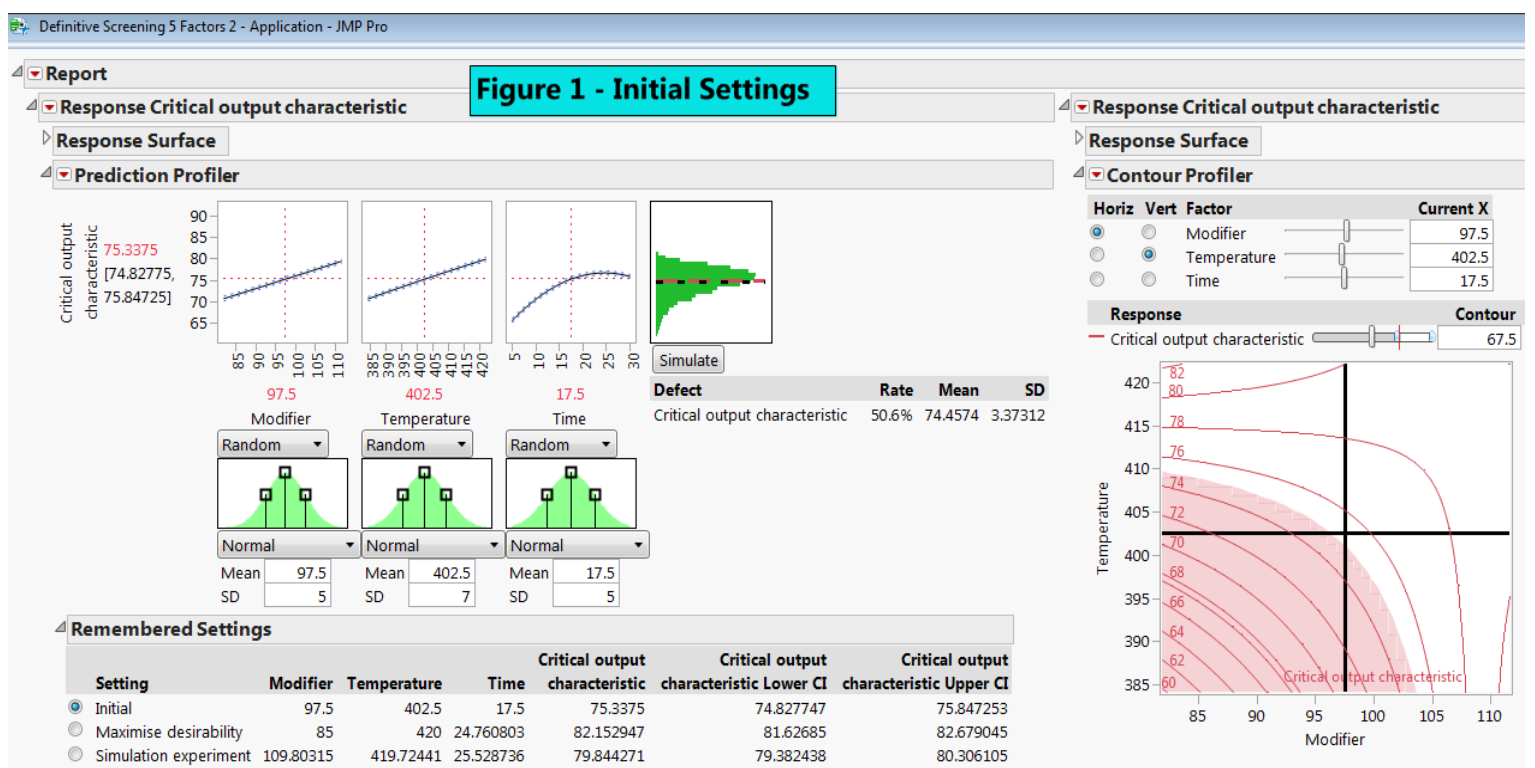

Figure 1. Initial settings for the critical factors

The Monte Carlo simulation randomly selects a value for each input factor from its proposed control distribution and substitutes these values into the multiple regression model to give a predicted value for the response. A random error is added to the predicted value for the response to represent the uncontrolled variation which is estimated from the variation in the regression model residuals. This additional component of variation represents variation due to uncontrollable factors or factors yet to be identified. For the response, the random error is simulated from a normal distribution with a mean of zero and a standard deviation estimated from the residuals of the regression model. Running a large number of such simulations (in this case 5000) gives an estimate of the defect rate (or out of specification rate) for the response and an overall defect rate when there is more than just one response.

Figure 1 shows that with the initial factor settings (the mid-point of the three-dimensional factor space), Critical output 
characteristic is predicted to have a value of $75.3 \%$, and half the points from the Monte Carlo simulation are below the lower spec limit. The contour plot shows that for these initial factor settings the process is running almost exactly on the lower spec limit (regions of the factor space where output values fall below the spec limit of $75 \%$ are shaded).

Using a desirability function and a steepest ascent search algorithm, the factor space can be explored to determine the factors settings that give the highest possible predicted value for the Critical output characteristic. Figure 2 shows that with the settings determined by JMP's "maximise desirability" option, Critical output characteristic increases to $82.1 \%$ and the proportion of points below the lower spec limit drops to $4.6 \%$. The contour plot shows that the process with those maximise desirability settings is now operating in the top left-hand corner where the highest value of the Critical output characteristic is predicted to be.

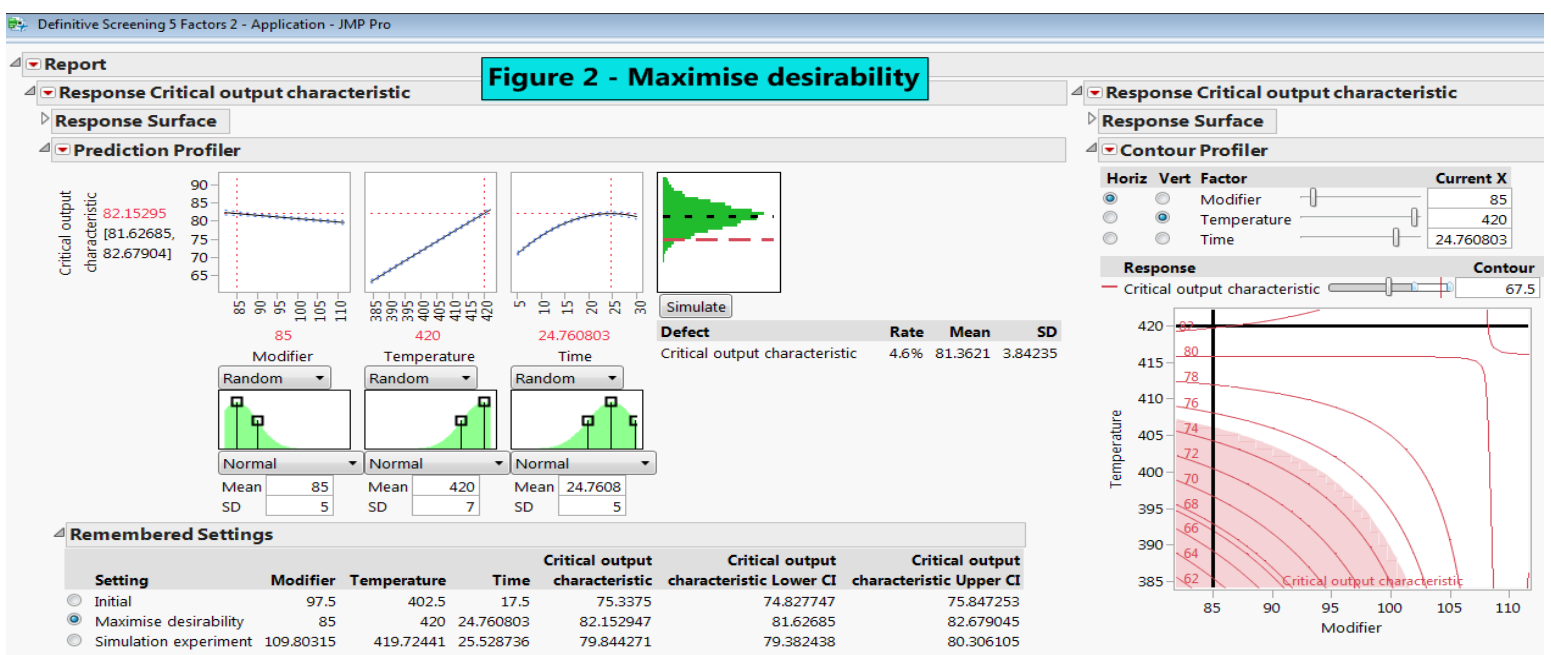

Figure 2. Factor settings obtained using maximise desirability on the response mean

\subsection{Process Improvement Using Monte Carlo Simulation Experiments}

While maximise desirability did find improved settings for the critical factors and succeeded in reducing the defect rate, this method only takes account of how the mean of the predicted response changes within the factor space. It doesn't take any account of how the variability in the factor values propagates to variation in the response. To properly take account of this variability in the inputs, Monte Carlo simulation experiments need to be run to explore the factor space in a different way. Rather than searching the factor space for the settings that give the most desirable mean value for Critical output characteristic, the simulation experiment approach focusses instead on searching the factor space to find the settings that minimise the calculated defect rate. The simulation experiment still uses the same polynomial model as maximise desirability, but now uses that model to run a series of Monte Carlo simulations to determine how the defect rate varies within the factor space. The simulation experiment uses a space filling design to explore the factor space and models the resulting defect rates using a Gaussian process. A space filling design is used to efficiently explore the factor space. Pronzato and Müller (2012) critique this approach and propose further refinements. A Gaussian process is used because the model needs to precisely fit the values obtained from the Monte Carlo simulations, and interpolate smoothly between these. Details of this method are provided in the JMP Profilers guide by SAS Institute (2015).

JMP provides some options for how the space filling design is constructed, and once all runs are completed, a table is generated. The results in the table are simply analysed using scripts that are automatically saved with the table; Table 2 shows the results from a 128 run simulation experiment.

Table 2. Results from 128-run simulation experiment and prediction profiler for Log (defect rate)

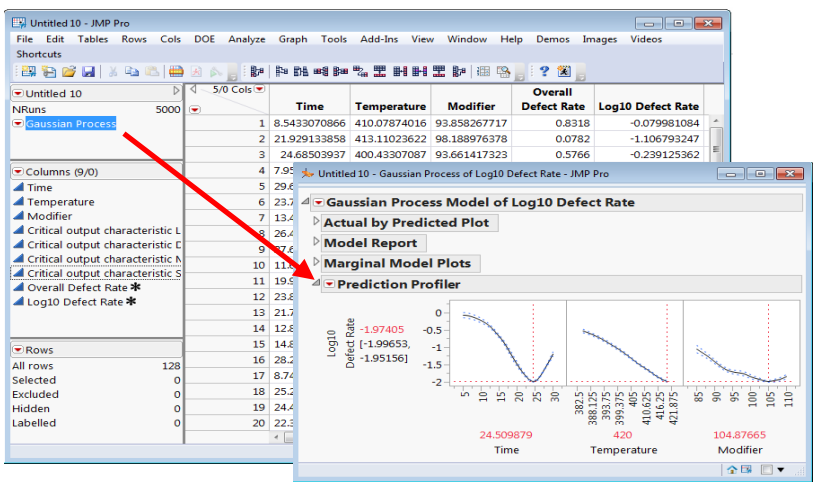


Each row in Table 2 represents a Monte Carlo simulation run with different factor settings. The log (defect rate) results are modelled using a Gaussian process and Table 2 also shows the prediction profiler obtained for the log (defect rate) model. Using the maximise desirability option, the optimum settings that minimise the log (defect rate) can be obtained. These optimum settings can then be used in the prediction profiler for Critical output characteristic and new Monte Carlo simulations can be run at this point to confirm the expected defect rate.

The settings determined by the simulation experiment position the process at the top right-hand corner of the contour plot, where the contour lines are farther apart. Figure 3 shows that these optimum settings predict a slightly lower value for Critical output characteristic $(79.8 \%)$. However, the proportion of points below the lower spec limit is now substantially reduced to $1.8 \%$ (compared to the $4.6 \%$ obtained with maximise desirability on the mean predicted response). When we compare the input settings for the maximise desirability solution to the simulation experiment solution, we can see that the main difference is that the simulation experiment has chosen a high setting for Modifier, which exploits the two-way interaction between Modifier and Temperature. This makes Critical output characteristic insensitive to changes in Temperature (the Temperature line in the Profiler is now flat). The predicted distribution of Critical output characteristic is much tighter with considerably fewer points below the lower specification limit, resulting in a more robust process.

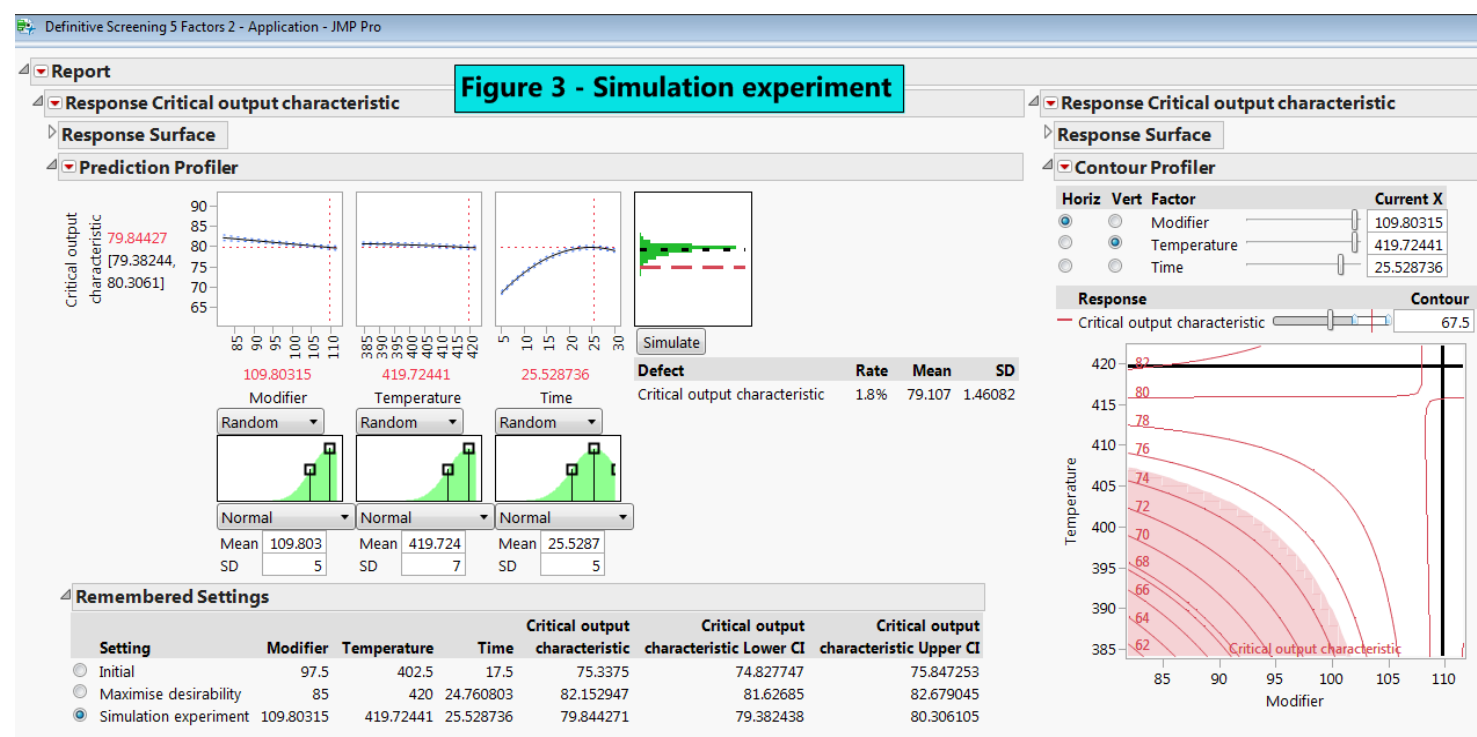

Figure 3. Factor settings obtained using Monte Carlo simulation experiment on the defect rate

\subsection{The Advantage of the Simulation Experiment}

The key difference between maximise desirability using the response mean and the simulation experiment approach using the defect rate is that the former doesn't take account of the unavoidable variation in the factors when choosing their settings, whilst the simulation experiment approach does. Consequently the simulation experiment is able to find more robust process settings that will minimise the variation in the critical output and reduce the overall defect rate.

\section{Conclusion}

Design of Experiments (DoE) is often used to investigate how the mean response of a process performance characteristic is affected by the settings of the input variables. Tolerance analysis is different in that it investigates how the variability in process performance is affected not just by the set points for each input but also by the unavoidable variability about these settings. As shown in the case study in this paper, these different objectives often lead to different "optimal" process settings. When faced with real-world situations, tolerance analysis is generally of more value because it yields greater process understanding, resulting in better and more cost-effective operational decisions about process management.

The simulation experiment approach described here provides a straightforward way of performing tolerance analysis that engineers can use without the need for an extensive background in statistical methods. Coupled with definitive screening designs, it provides all the benefits of robust process engineering promised by Taguchi but with far less investment of resources, cost and time. 


\section{References}

Box G. E. P., Hunter, J. S., \& Hunter, W. G. (2005). Statistics for Experimenters: Design, Innovation, and Discovery $\left(2^{\text {nd }}\right.$ ed.). New York, NY: Wiley.

Goos, P., \& Jones B. (2011). Optimal Design of Experiments: A Case Study Approach. New York, NY: Wiley.

Jones, B., \& Nachtsheim, C. J. (2011) A Class of Three-Level Designs for Definitive Screening in the Presence of Second-Order Effects. Journal of Quality Technology, 43(1), 1-15

Kenett, R., \& Zacks, S. (2014). Modern Industrial Statistics: with Applications in R, Minitab and JMP (2 ${ }^{\text {nd }}$ ed.). New York, NY: Wiley.

KPA Group. (2015). The QbD Column: Overview of Quality by Design. Retrieved from http://blogs.sas.com/content/jmp/2015/05/07/the-qbd-column-overview-of-quality-by-design/

Monte Carlo method. (2015). In Wikipedia, the free encyclopaedia. http://en.wikipedia.org/wiki/Monte_Carlo_method

Montgomery, D. C. (2012). Design and Analysis of Experiments JMP ( $8^{\text {th }}$ ed.). New York, NY: Wiley.

Moore, M., Anderson, R., \& Kay, P. (2015). Optimising Processes with Design of Experiments, http://www.jmp.com/doecasestudies

Pronzato, L., \& Müller, W. (2012), Design of computer experiments: space filling and beyond, Statistics and Computing, 22, 681-701, http://dx.doi.org/10.1007/s11222-011-9242-3

Rubinstein, R. Y., \& Kroese, D. P. (2008) Simulation and the Monte Carlo method. New York, NY: Wiley.

SAS Institute Inc. (2015). JMP® 12 Profilers. Cary, NC: SAS Institute Inc.

Wei, Z., Moore, M., \& Kussener, F. (2013) Visual Tolerance Analysis for Engineering Optimisation. International Journal of Metrology and Quality Engineering, 4(3), 153-162, http://dx.doi.org/10.1051/ijmqe/2013056

\section{(cc) $\mathbf{E Y}$}

This work is licensed under a Creative Commons Attribution 3.0 License. 